\title{
GCU
}

Glasgow Caledonian

University

University for the Common Good

\section{Adding rotation to translation: percepts and illusions}

Magnussen, Camilla M.; Orbach, Harry S.; Loffler, Gunter

Published in:

Perception

DOI:

$10.1068 / p 7739$

Publication date:

2014

Document Version

Author accepted manuscript

Link to publication in ResearchOnline

Citation for published version (Harvard):

Magnussen, CM, Orbach, HS \& Loffler, G 2014, 'Adding rotation to translation: percepts and illusions', Perception, vol. 43, no. 9, pp. 926-946. https://doi.org/10.1068/p7739

\section{General rights}

Copyright and moral rights for the publications made accessible in the public portal are retained by the authors and/or other copyright owners and it is a condition of accessing publications that users recognise and abide by the legal requirements associated with these rights.

Take down policy

If you believe that this document breaches copyright please view our takedown policy at https://edshare.gcu.ac.uk/id/eprint/5179 for details of how to contact us. 


\section{Adding Rotation to Translation: Percepts and Illusions}

Camilla M. Magnussen ${ }^{1}$, Harry S. Orbach ${ }^{1}$ and Gunter Loffler ${ }^{1}$

${ }^{1}$ Department of Life Sciences, Glasgow Caledonian University, Cowcaddens Road, Glasgow G4 0BA,UK

E-mail: $\quad$ CMM: cmagnussen@gnotometrics.com

HSO: H.Orbach@gcu.ac.uk

GL: G.Loffler@gcu.ac.uk

Corresponding authors:

Camilla M. Magnussen ${ }^{a}$

Gunter Loffler

Phone: $\quad$ +441413313386

Acknowledgements: This research was supported in part by EPSRC Grant No. GR/S59239/01.

a Present address: GN Otometrics, Hørskætten 9, 2630 Taastrup; Denmark 


\section{Abstract}

This study investigated how the perception of a translating object is affected by rotation. Observers were asked to judge the motion and trajectory of objects that rotated around their centroid while linearly translating.

The expected percept, consistent with the actual dynamics used to generate the movie sequences, is that of a translating and rotating object, akin to a tumbling rugby ball. Observers, however, do not always report this and, under certain circumstances, perceive the object to translate on an illusory curved trajectory, similar to a car driving on a curved road. The prevalence of veridical versus non-veridical percepts depends on a number of factors. First, if the object's orientation remains within a limited range relative to the axis of translation, the illusory, curved percept dominates. If the orientation, at any point of the movie sequence, differs sufficiently from the axis of translation, the percept switches to linear translation with rotation. The angle at which the switch occurs is dependent upon a number of factors that relate to an object's elongation and, with it, the prominence of its orientation. For an ellipse with an aspect ratio of 3, the switch occurs at approximately $45 \mathrm{deg}$. Higher aspect ratios increase the range, lower ratios decrease it. This applies similarly to rectangular shapes. A line is more likely to be perceived on a curved trajectory than an elongated rectangle, which, in turn, is more likely seen on a curved path than a square. This is largely independent of rotational and translational speeds.

Measuring perceived directions of motion at different instants in time allows the shape of the perceived illusory curved path to be extrapolated. This results in a trajectory that is independent of object size and corresponds closely to the actual object orientation at different points during the movie sequence.

This provides evidence for a perceptual bi-stability in the form of a transition from an illusory curved trajectory to a veridical linear trajectory (with rotation) for the same object. Both are consistent with special real-world cases such as objects rotating around a centre off the object so that their orientation remains tangent to the trajectory (cheetahs running along a curve, sailboats) or objects tumbling along simple trajectories (a monkey spinning in air, spinning cars on ice). In the circumstances shown here, the former is an illusion.

Keywords: motion illusion, complex motion, rotational motion, translational motion, object motion, object orientation 


\section{Introduction}

When the visual system samples motion information from a dynamic environment, it will typically be confronted with local estimates that are inconsistent with each other. An obvious scenario is when multiple objects move in different directions. In this case, the task for the visual system is to decide which of the samples belong to the same object and combine them while segregating information from other objects. This cannot simply be solved by combining those estimates that share the same motion, even for the simplest case of a rigid object translating linearly. The locally sampled motion signals depend on the position on the object from which they are taken. Features on the object (e.g. the corner of a translating diamond) yield veridical motion information but contours (the diamond's sides) are ambiguous and consistent with an infinite number of possible motions (the aperture problem, Wallach, 1935). A considerable number of studies have investigated how the visual system deals with multiple, different motion estimates (Hildreth, 1984; Adelson \& Movshon, 1982; Ferrera \& Wilson, 1990; Weiss \& Simoncelli, 2002), typically concentrating on rigid translation and often employing superimposed gratings (Adelson \& Movshon, 1982; Ferrera \& Wilson, 1990; Wilson et al., 1992). Models were derived that predict when signals are combined into a uniform percept or perceived as moving independently as in the case of transparency (Wilson et al., 1992; Adelson \& Movshon, 1982).

Others have looked at the role of object features in the process of signal integration given that tracking one of its features can directly derive the veridical motion of a translating object. It has been shown that the visual system indeed relies heavily on features if they 
are present (Hildreth, 1984; Nakayama \& Silverman, 1988; Lorenceau \& Shiffrar, 1992; Grossberg \& Mingolla, 1993; Loffler \& Orbach, 2001, Loffler \& Orbach, 2003a) and models have been proposed to account for the capturing effect of features on signals from other regions of a rigidly translating object (Loffler \& Orbach, 2003b, Park et al., 2004).

All of these studies have considered the relatively simple case of rigid translation. Others have looked at rigid rotation (Kennedy, Orbach, Gordon \& Loffler, 2008). Here we aimed to investigate the question of how the visual system deals with a single, rigid object undergoing rotation plus translation. In such cases, the motion signals do not only depend on their locations on the object, they also change over time. The stimuli we employed here were objects that translated linearly while rotating around their centre of gravity (see Figs. $1 \& 9)$. A veridical percept is, therefore, e.g. a rotating ellipse moving horizontally, i.e. the two motions (rotation and translation) are perceived separately. An illusory percept would be that of an ellipse "sailing" along a curved trajectory. In this case, the visual system would incorrectly combine the two motions. In the latter case, the percept is akin to a car going around a curve, in the former it is similar to a spinning car $^{2}$.

There is reason to believe that such illusory percepts may arise based on earlier studies on rigid, linearly translating objects (Loffler \& Orbach, 2001; Magnussen, Orbach \& Loffler, 2013, Morikawa, 1999). Perception was shown to be biased for these stimuli, away from

\footnotetext{
${ }^{2}$ Note that there are an infinite number of veridical descriptions for such a case of constant motion (Yang et al., 2002). It can be shown that, for any motion in two dimensions, one can pick any one of a number of centres of rotation on the rigid body and express the motion as translation (often non-linear) of that centre and rotation around that centre. However, the percept of an object moving along a curved trajectory when it actually translates linearly (with or without rotation) is not one of the veridical descriptions and thus is illusory. We will return to this point in the discussion.
} 
veridical and typically towards the orientation or elongation/symmetry axis of the object. This lead to the speculation that, if there is a bias towards perceiving an object's direction of motion along its axis of elongation, one may perceive a linearly translating object as if it was moving along a curved trajectory if the object changes orientation (i.e. rotates) while translating (Fig. 1). As the results will show, this illusory percept is indeed what observers report under certain circumstances. If these circumstances are not met, the percept is that of separate translation and rotation.

\section{METHODS}

\subsection{STIMULI}

A range of shapes, as depicted in Fig. $1(\mathrm{~A})$, were set to translate with a velocity $V_{T}$ along a specified direction and to rotate with a rotational speed $V_{R}$ relative to a centre of rotation. Although the absolute translational and rotational speeds varied across conditions, rotation and translation were always kept constant during each presentation. Translation was always linear and rotation always counter-clock-wise, CCW, around the centroid (centre of mass, geometric centre) of each shape. In some experiments the axis of translation was along the horizontal and in others along the diagonal.

\subsection{PROCEDURES}

Two different procedures were used in the experiments. The first (Fig. 1 C) determined the circumstances under which observers perceive objects to move along a curved or a straight trajectory. The second procedure (Fig. $1 \mathrm{D}$ ) was used to measure the perceived trajectory in detail for conditions where observers reported a curved path. 


\subsubsection{PROCEDURE 1}

Six frames of a sample movie sequence are shown in Fig. 1 (B) for the case of an ellipse rotating around its centre while translating along the horizontal to the right $\left(0^{\circ}\right.$ corresponds to the right and $+90^{\circ}$ to vertically upward). In different trials, the movies were terminated after a different number of frames in order to measure perception (curved versus straight trajectory) as a function of presentation time. Different movie lengths were randomly intermixed within an experimental block.

In order to familiarise the observers with the stimuli, they were presented with two demonstrations prior to the actual experiments (similar to supplementary material). Both demonstrations used identical ellipses that differed in translational and rotational speed and overall presentation time. Following each demonstration, observers were asked to describe the motion they perceived. For all participants, the first demonstration was perceived as an ellipse translating smoothly along a curved trajectory, consistent with a car driving around a corner. This differed from the second demonstration, where observers reported seeing a rotating ellipse translating linearly. This latter percept was described as a spinning car. 
(A) Stimuli

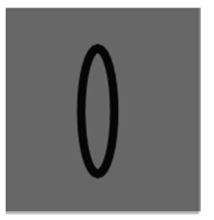

$\mathrm{a}=2 / 3^{\circ}, \mathrm{b}=2^{\circ}$

profile $=0.25^{\circ}$

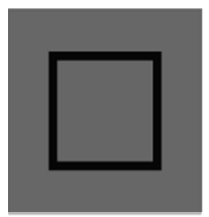

$\mathrm{a}=2^{\circ}, \mathrm{b}=2^{\circ}$

profile $=0.25^{\circ}$

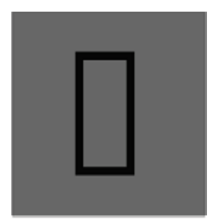

$\mathrm{a}=1^{\circ}, \mathrm{b}=2^{\circ}$

profile $=0.25^{\circ}$

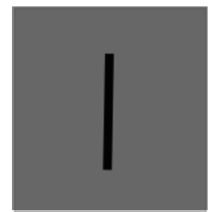

length $=4^{\circ}$

profile $=0.25^{\circ}$
(B) Motion

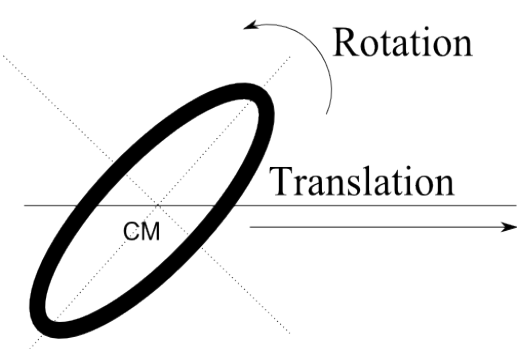

Centre of mass $(\mathrm{CM})$ on axis of translation

(C) Procedure 1: Is the perceived trajectory curved (1) or straight (2)?

Motion sequence:

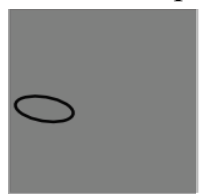

$-10^{\circ}$

0s

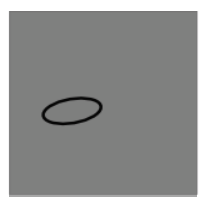

$10^{\circ}$

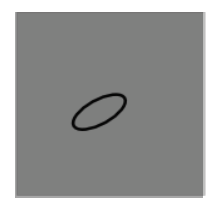

$30^{\circ}$

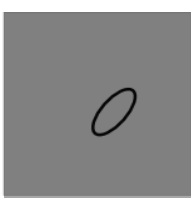

$50^{\circ}$

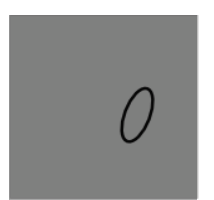

$70^{\circ}$

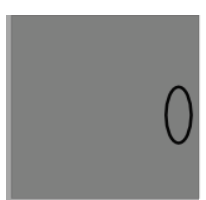

$90^{\circ}$

$0.9 \mathrm{~s}$

$1.2 \mathrm{~s}$

$1.5 \mathrm{~s}$

Time (sec)

Percept:

(1) Curved trajectory

(2) Straight trajectory
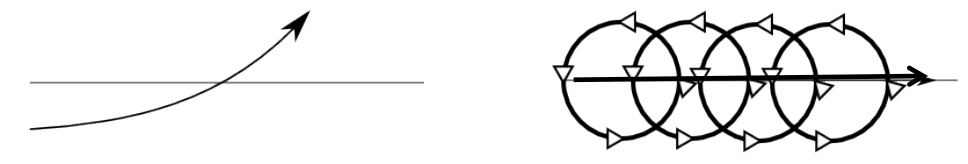

(D) Procedure 2: What is the current direction of motion?

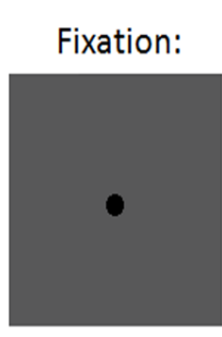

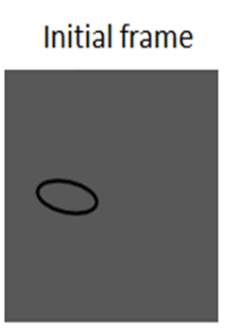

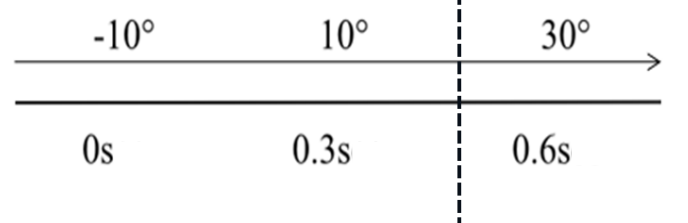

Respond:

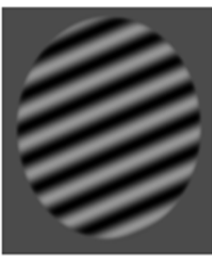

Is the current direction of motion $\mathrm{CW}$ or CCW to the grating's orientation? 
Figure 1: (A) Examples of stimuli used. The movies employed a range of contour shapes that rotated while translating. The aspect ratios of the shapes were defined by two parameters ("a" and "b") corresponding to half their width and length. The examples in (A) all show shapes of $4^{\circ}$ length. The profile (cross-section) of the shapes was set to $0.25^{\circ}$ and identical for all stimuli. (B) Stimulus motion. The example shows an ellipse linearly translating to the right along the horizontal axis so that its centroid (centre of mass, CM) remains on the horizontal. While translating, the ellipse rotates around the CM. (C) Example of a movie sequence for the case of an ellipse translating linearly along the horizontally and rotating CCW. The ellipse is initially (0s) oriented at $10^{\circ}$ relative to the horizontal. For the depicted rotational speed of $66.7 \mathrm{deg} / \mathrm{s}$, the ellipse' orientation is at $+10 \mathrm{deg} 300 \mathrm{~ms}$ after the start of the movie and at $+90 \mathrm{deg}$ at $1500 \mathrm{~ms}$. The movie sequence was stopped at different points in time and the observers were asked to indicate whether they perceived the entire sequence as (1) an object translating along a curved trajectory or (2) an object translating along a straight trajectory while rotating. (D) In a separate experiment, the perceived direction of motion at different instants of time was measured. The example shows again the case of rotating and horizontally translating ellipse. At the start of the sequence, a fixation point was presented at the centre of the screen. The movie was symmetric with respect to the fixation point so that for the example shown the ellipse first appeared to the left of the fixation point and moved to the right, crossing the centre of the screen halfway through the sequence. The observers' task was to indicate the perceived direction of motion at the point where the movie sequence was terminated (instantaneous direction of motion). After the last frame (indicated with a surrounding dashed rectangle), a stationary grating appeared and observers had to report whether the perceived direction of motion was $\mathrm{CW}$ or $\mathrm{CCW}$ relative to the grating's orientation. The orientation of the grating was altered and $\mathrm{CW} / \mathrm{CCW}$ responses used to determine the point of subjective equality. Different sequence lengths were used to determine the perceived direction of motion at different times of the movie.

For the subsequent experiments, the two types of percepts were labelled (1) translation on a curved trajectory and (2) translation on a straight trajectory, and observers were instructed to indicate their percept using the keyboard. This task is similar in spirit to those used in transparency studies where observers have to report if they "see coherence" or "see transparency" (Kim \& Wilson, 1994). 


\subsubsection{PROCEDURE 2}

The second procedure was designed to describe the perceived trajectory in more detail in those circumstances where observers perceived a rotating and translating object as following a curved trajectory. In order to do that, tangents (instantaneous perceived directions of motion) to the perceived trajectory were measured at different points in time. The entire trajectories were then extrapolated from these measurements using a $1^{\text {st }}$ order integration method (Euler integration) under the assumption that the trajectories are increasing monotonically (for justification, see below).

The perceived directions of motion were measured using a 2AFC paradigm where observers had to report whether the motion of an object was clockwise $(\mathrm{CW})$ or counterclockwise $(\mathrm{CCW})$ relative to the orientation of a static grating. The durations of the movie sequences were randomly intermixed and observers asked to report the perceived direction of motion at the point of the last movie frame. Perceived directions were defined as the point of subjective equality (PSE), where observers reported the motion direction to be $\mathrm{CW}$ and $\mathrm{CCW}$ relative to the grating's orientation with equal probability. The procedure is illustrated in Fig. 1D. In order to appropriately capture the PSE, the orientations of the static reference gating were set individually, depending on stimulus details and observer sensitivity.

\subsection{APPARATUS}

The stimuli were presented on a gamma-corrected LaCie electron blue 22" (mean luminance $=75 \mathrm{~cd} / \mathrm{m}^{2}$ ) monitor driven by an Apple Macintosh $\mathrm{G} 4$. The frame refresh rate of the monitor was set to $85 \mathrm{~Hz}$ and the spatial resolution to $1024 \times 768$ pixels. A chin and 
forehead rest was used to maintain a constant viewing distance of $80 \mathrm{~cm}$. Each pixel subtended 0.0266 dva at this distance. To avoid reference cues, the monitor frame was covered with a white cardboard mask with a circular aperture subtending $13.5 \mathrm{dva}$. The movies were based on a sequence of frames $(11.76 \mathrm{~ms})$, each calculated independently and prior to the experiments. The programs employed routines from Pelli's Videotoolbox (Pelli, 1997).

\subsection{OBSERVERS}

A total of seven observers participated in the experiments. Six of those completed the first condition, which examined the general percept. Four of them were naïve as to the purpose of the study. The perceived shape of the trajectory, was measured on three of the seven observers, one of them naïve. All observers had normal or corrected-to-normal vision.

\subsection{DATA ANALYSIS}

The data obtained from procedure 1 and 2 were fitted with a Quick function (Quick, 1974) using a maximum-likelihood procedure and the point of subjective equality (PSE), where observers are equally likely to report the two precepts, was defined as the $50 \%$ point on the fitted function. $95 \%$ confidence intervals were obtained using bootstrap analysis provided by the Palamedes toolbox (Prins \& Kingdom, 2009).

When determining the shape of the perceived trajectory, PSEs for the perceived direction of motion were measured for different movie durations. $A 1^{\text {st }}$ order Euler method was then used to reconstruct the perceived curve from the measured directions (tangents to the 
trajectory). From any point on a curve, one can find an approximation of a nearby point by moving a short distance (h) along the tangent $(\mathrm{dy} / \mathrm{dx})$ to the curve:

$y(x+h) \approx y(x)+h \cdot y^{\prime}(x)$

where $\mathrm{h}$ is the step size, i.e. the distance between two successive measurement points.

Each subsequent point on the curve is found by multiplying the tangent with the step size and adding it to the current position. The trajectory cannot be determined without ambiguity as the overall position of the curve depends on a starting position. We chose the initial starting point to be veridical, i.e. at the position of the ellipse's centre at time 0 $(x(0)=-2.1 d v a$ and $y(0)=0 \mathrm{dva})$. Other starting points could have been chosen but would not have changed the extrapolated shapes of the trajectories. 


\section{RESULTS}

\subsection{EXPERIMENT 1: EFFECT OF FINAL ELLIPSE ORIENTATION}

\begin{tabular}{|c|c|c|c|c|c|c|}
\hline & \multicolumn{5}{|c|}{ Fixed Parameters } & $\begin{array}{l}\text { Independent } \\
\text { parameter }\end{array}$ \\
\hline \multicolumn{7}{|c|}{ Experiment 1: Effect of final ellipse orientation } \\
\hline \multirow[t]{3}{*}{ (A) } & Translational & Rotational & Initial & \multicolumn{2}{|c|}{ Shape } & Final orientation \\
\hline & Speed & Speed (CCW) & Orientation & \multicolumn{2}{|c|}{$a \quad b$} & \\
\hline & $7 \mathrm{dva} / \mathrm{s}$ & $68 \% / s$ & $-10^{\circ}$ & $2 / 3$ dva & $2 d v a$ & $\begin{array}{l}0.4^{\circ} ; 14.8^{\circ} ; 20.4^{\circ} ; 25.2^{\circ} ; \\
30.0^{\circ} ; 34.8^{\circ} ; 40.4^{\circ} ; 45.2^{\circ} \text {; } \\
50.0^{\circ} ; 54.8^{\circ} ; 60.4^{\circ} ; 65.2^{\circ} ; \\
70.0^{\circ} ; 74.8^{\circ} ; 80.4^{\circ} ; 85.2^{\circ} ; \\
90^{\circ} \\
\text { Corresponding movie } \\
\text { length for } 90^{\circ} \text { is } 1.471 \mathrm{~s}\end{array}$ \\
\hline \multicolumn{7}{|c|}{ Experiment 2: Effect of rotational and translational speed } \\
\hline \multirow[t]{2}{*}{ (B) } & $\begin{array}{c}\text { Final } \\
\text { Orientation }\end{array}$ & $\begin{array}{c}\text { Rotational } \\
\text { Speed (CCW) }\end{array}$ & $\begin{array}{c}\text { Initial } \\
\text { Orientation }\end{array}$ & a & b & Translational speed \\
\hline & $20^{\circ}, 45^{\circ}, 90^{\circ}$ & $68^{\circ} / \mathrm{s}$ & $-10^{\circ}$ & $2 / 3$ dva & 2 dva & $2,3,7,15 \mathrm{dva} / \mathrm{s}$ \\
\hline \multirow[t]{3}{*}{ (C) } & $\begin{array}{c}\text { Final } \\
\text { Orientation }\end{array}$ & $\begin{array}{c}\text { Translational } \\
\text { Speed }\end{array}$ & $\begin{array}{c}\text { Initial } \\
\text { Orientation }\end{array}$ & a & $b$ & Rotational speed \\
\hline & $20^{\circ}, 45^{\circ}, 90^{\circ}$ & $7 \mathrm{dva} / \mathrm{s}$ & $-10^{\circ}$ & $2 / 3$ dva & $2 d v a$ & $\begin{array}{c}17,34,68,76,85,102 \\
116 \%\end{array}$ \\
\hline & \multicolumn{6}{|c|}{ Experiment 3: Effect of initial ellipse orientation } \\
\hline \multirow[t]{4}{*}{ (D) } & $\begin{array}{l}\text { Translational } \\
\text { Speed }\end{array}$ & $\begin{array}{c}\text { Rotational } \\
\text { Speed (CCW) }\end{array}$ & $\begin{array}{c}\text { Initial } \\
\text { Orientation }\end{array}$ & a & $\mathrm{b}$ & Final Orientation \\
\hline & $7 \mathrm{dva} / \mathrm{s}$ & $68 \%$ s & $10^{\circ}$ & $2 / 3$ dva & 2 dva & $35^{\circ}, 45^{\circ}, 55^{\circ}, 60^{\circ}$ \\
\hline & $7 \mathrm{dva} / \mathrm{s}$ & $68 \% / \mathrm{s}$ & $-35^{\circ}$ & $2 / 3$ dva & 2 dva & $10^{\circ}, 35^{\circ}, 45^{\circ}, 55^{\circ}$ \\
\hline & $7 \mathrm{dva} / \mathrm{s}$ & $68 \% / \mathrm{s}$ & $-90^{\circ}$ & $2 / 3$ dva & $2 \mathrm{dva}$ & $-35^{\circ},-20^{\circ},-10^{\circ}, 10^{\circ}$ \\
\hline \multicolumn{7}{|c|}{ Experiment 4: Effect of shape: aspect ratio } \\
\hline \multirow[t]{2}{*}{ (E) } & $\begin{array}{c}\text { Initial } \\
\text { Orientation }\end{array}$ & $\begin{array}{c}\text { Final } \\
\text { Orientation }\end{array}$ & $\begin{array}{l}\text { Translationa } \\
\text { I Speed }\end{array}$ & \multicolumn{2}{|c|}{$\begin{array}{l}\text { Rotational } \\
\text { speed }\end{array}$} & Aspect ratio \\
\hline & $-10^{\circ}$ & $25^{\circ}$ & $7 \mathrm{dva} / \mathrm{s}$ & \multicolumn{2}{|c|}{$68 \% \mathrm{~s}$} & $1,1.2,1.5,1.7,2,3,12$ \\
\hline
\end{tabular}

Table 1: The parameters used in the experiments. (A) Effect of final ellipse orientation. Terminating the movie sequence at different points in time results in a range of ellipse orientations in the final frame as shown in the right hand column. The final orientations ranged from 0.4 to $90 \mathrm{deg}$ (vertical). (B \& C) A range of translational and rotational speeds was used for three final ellipse orientations (20,45 and 90deg). Rotation was always counter-clockwise. (D) Effect of initial orientation. Three different initial orientations were used, each with a range of final orientations. 
(E) Effect of aspect ratio. Ellipses with different aspect ratios were used with initial and final orientations that showed a curved trajectory in (A) and (D).

In the first experiment, observers were asked to report if the perceived trajectory of a rotating and translating ellipse was straight or curved. The parameters for this condition are shown in Table $1 \mathrm{~A}$. The initial orientation of the ellipse was -10deg relative to the horizontal (see Fig. $1 \mathrm{C}$ ). The movie sequence was terminated at different points in time. Data (Fig. 2) are presented as the percentage of times the observers reported a curved trajectory as a function of the orientation of the ellipse in the final frame, which co-varied with presentation time. The transition point (PSE), where observers are equally likely to see a curved and a straight trajectory, for each of the 6 observers for translation along the horizontal (Fig. 2) is shown (with $95 \% \mathrm{Cl}$ ) next to the individual data.

The results show that a rotating and translating ellipse can be perceived either as moving on a straight or curved trajectory, depending on the final orientation of the ellipse. The first percept is consistent with the movie sequence, the second is an illusion: an ellipse, which is translating linearly, is perceived to follow a curved path. The average PSE was $45.3 \mathrm{deg}$ (range: $23-63^{\circ}$ ). If the final orientation was below that value, observers were more likely to report a curved trajectory, if it was above, they more often reported a straight trajectory.

In order to investigate if the orientation at which the percept switched showed a dependence on the absolute orientation of the ellipse, we repeated the experiment for a diagonal trajectory $(+45 \mathrm{deg}$, i.e. up and to the right, Fig. 3). All other parameters were identical, including translational and rotational speeds as well as initial and final ellipse orientations, although those were now relative to the diagonal axis of translation. The data 
for the diagonal show little difference to those for the horizontal. The average PSE is 42.6deg relative to the diagonal (absolute orientation of $87 \mathrm{deg}$ ). A two sample, two-tailed $\mathrm{t}$-test $(\mathrm{t}=0.71, \mathrm{df}=16, p=0.48)$ indicates that there is no significant difference between horizontal and diagonal axes of translation. This suggests that perception does not depend on the translational axis, nor does it depend on the absolute final orientation of the ellipse. Rather, the orientation of the ellipse relative to the axis of translation determines if observers perceive a straight or curved trajectory.

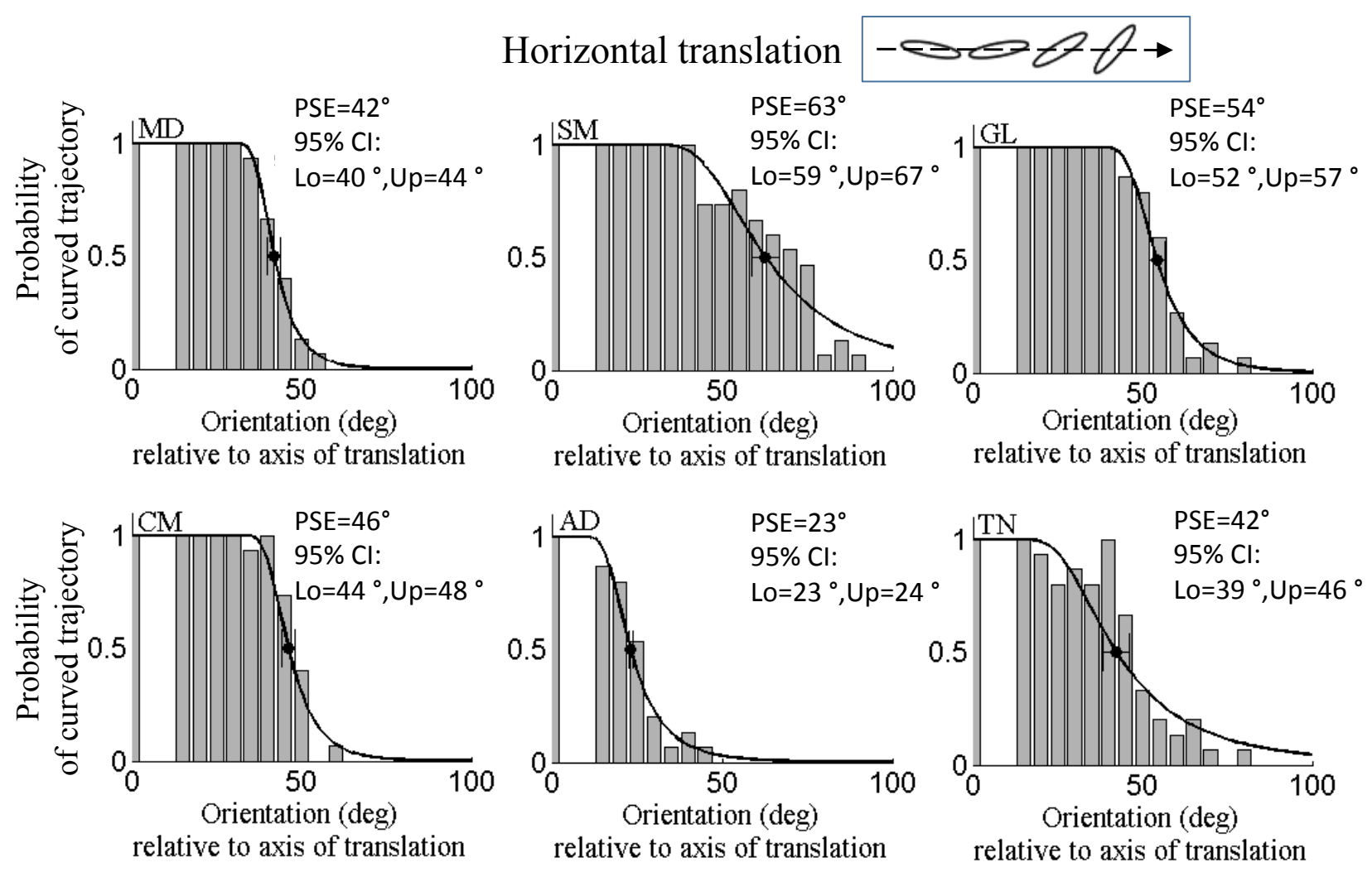

Figure 2: Perception of a rotating ellipse translating along the horizontal. Data ( $\mathrm{N}=6$, grey bars) show the probability of perceiving a (illusionary) curved trajectory as a function of the final ellipse orientation. The black lines show the fitted psychometric functions. PSE (50\%, i.e. equally likely to report curved and straight trajectory) and $95 \% \mathrm{Cl}$ are given for each individual observer (Lo=lower 
and Up=upper limits of the $\mathrm{Cl}$ ). Observers are more likely to perceive a curved trajectory when the final ellipse orientation stays, on average, below $45 \mathrm{deg}$ (stdev $\pm 13 \mathrm{deg}$ ).
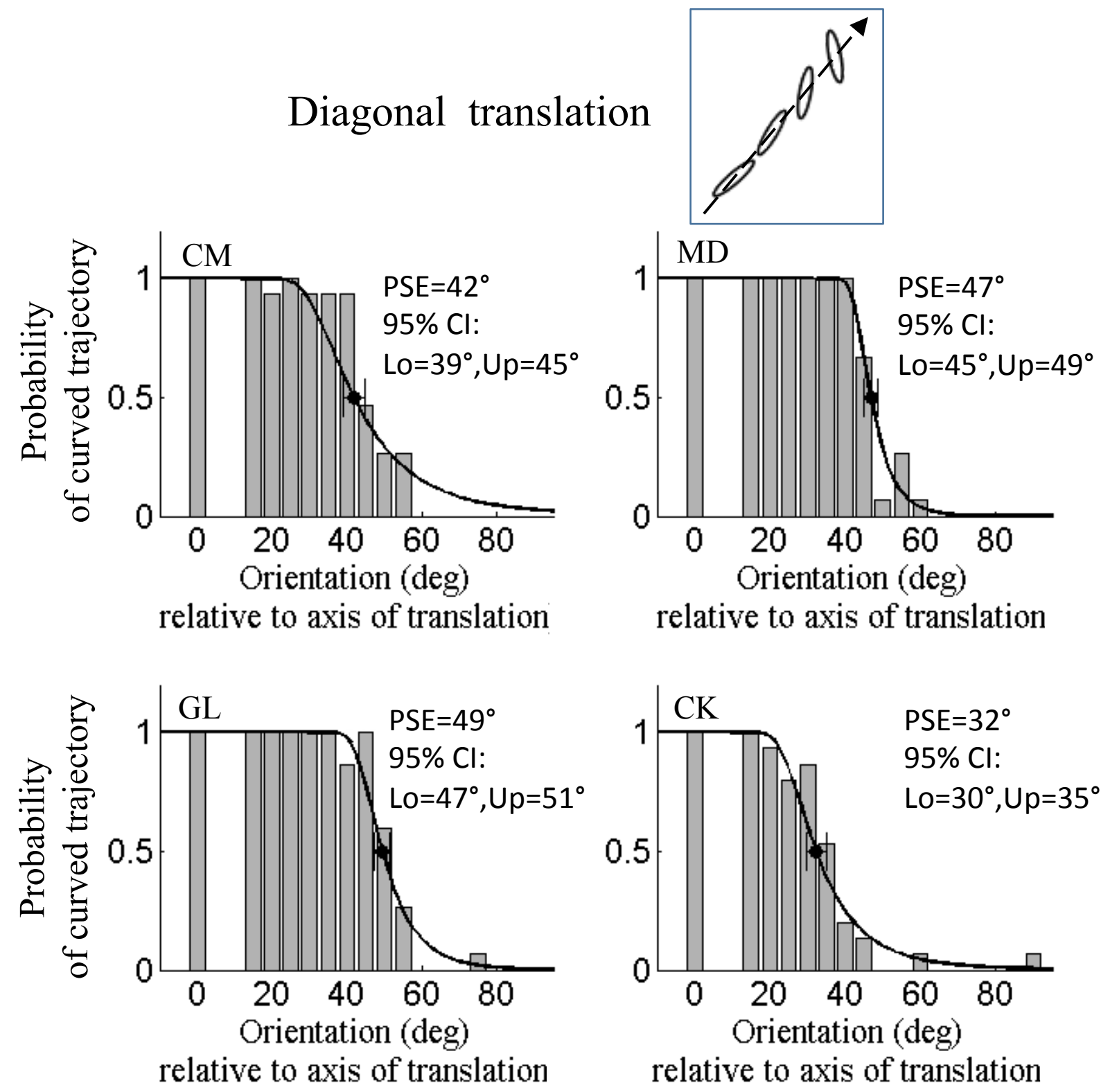

Figure 3: Perception of a rotating ellipse translating along the diagonal. Data (relative to the diagonal) for four observers are similar to those for the horizontal direction (average $P S E=42.6 \mathrm{deg} \pm 13$ ), suggesting that percepts depend primarily on the orientation of the ellipse relative to the trajectory and are largely independent of the absolute ellipse orientation and the axis of translation. 


\subsection{EXPERIMENT 2: EFFECT OF ROTATIONAL AND TRANSLATIONAL SPEED}

The first experiment suggested that percepts depend on the orientation of the ellipse relative to the trajectory. This does, however, co-vary with presentation time. This covariation can be broken in several ways. Varying translational speed, for each of three fixed final orientations, varies the presentation time. Varying rotational speed also allows one to distinguish between the two factors. If the percept depended on presentation time, just varying rotational speed should not affect the result. If it depended on the final orientation of the ellipse, the overall percept should remain unchanged when rotational speed and movie duration co-vary.

In the first part, the translational speed varied. The rotational speed and the initial orientation were kept constant. The final orientation was set to one of three values $\left(20^{\circ}\right.$, $45^{\circ}$ and $90^{\circ}$ ), which required that the presentation time co-varied with the translational speed (Table $1 \mathrm{~B}$ ). The final orientations were selected based on the result in the first experiment where the percept of translation on a curved trajectory was predominantly seen for final orientations of $20^{\circ}$, was equally likely as a straight trajectory for $45^{\circ}$ and generally absent for $90^{\circ}$. In the second part, the translational speed was fixed at $7^{\circ} / \mathrm{s}$ and the rotational speed varied (Table $1 \mathrm{C}$ ). As above, measurements were taken for final orientations of $20^{\circ}, 45^{\circ}$ and $90^{\circ}$. This required that presentation times co-varied with rotational speed.

The probability for perceiving a curved trajectory for the three final ellipse orientations $\left(20^{\circ}\right.$, $45^{\circ}$ and $90^{\circ}$ ) for the two conditions are shown in Fig. 4 . It is evident that the data for each 
of the final orientations show little dependence on translational (Fig. 4A) or rotational speed (Fig. 4B).
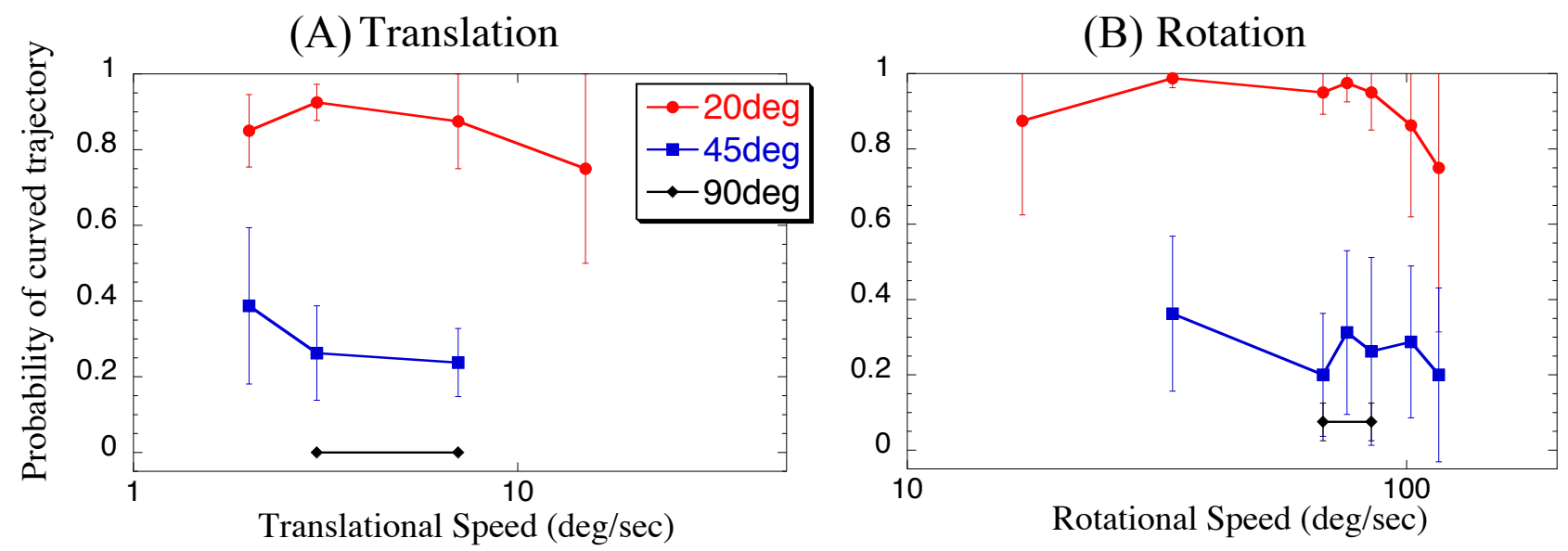

Figure 4: Effect of rotational and translational speed. The data show the average results for four observers who each repeated every condition 10 times. (A) Translational speed variation. If the final ellipse orientation is fixed at $20^{\circ}$ (red), a curved trajectory was perceived in approximately $85 \%$ of the trials largely independent of translational speed. Probabilities are on average $30 \%$ when the final orientation is $45^{\circ}$ (blue). For a final orientation of $90^{\circ}$ (black), observers never reported a curved trajectory. (B) Rotational speed variation. The probability of perceiving a curved trajectory for a final ellipse orientation of $20^{\circ}$ is on average $91 \%$, ranging from $75 \%$ to $98 \%$. For a final ellipse orientation of $45^{\circ}$, the likelihood of seeing a curved trajectory is $27 \%$ with little variation for different rotational speeds. For a final orientation of $90^{\circ}$, a curved trajectory was rarely perceived. Perceiving a rotating and translating ellipse as moving on a straight line or a curved trajectory is therefore largely independent of the translational and rotational speeds.

For the entire range of translational speeds (Fig. 4A), the probability of seeing a curved trajectory is $85 \%$ when the final ellipse orientation is $+20 \mathrm{deg}$ (presentation time of $441 \mathrm{~ms}$ ). When the final ellipse orientation is $45^{\circ}(808 \mathrm{~ms})$, the average probability of observing a curved trajectory is $30 \%$. Finally, observers always reported a straight trajectory ( $0 \%$ probability) when the final ellipse orientation is $90^{\circ}$. These results correspond well with those obtained in the first experiment and suggest that perception is relatively unaffected 
by translational speed, perhaps with the exception of the fastest translational speed (15dva/s) where the transition from curved to straight percept seems to occur at smaller final ellipse orientations.

Varying rotational speed, the probability of perceiving a curved trajectory is, on average, $91 \%$ for final ellipse orientations of $20^{\circ}$ (Fig. 4B). When the final orientation is $45^{\circ}$, the probability decreases to $27 \%$, and it is essentially absent for $90^{\circ}$.

In summary, it can be concluded that perceiving a rotating and translating ellipse as moving on a straight line or a curved trajectory is largely independent of the translational and rotational speeds because results are unaffected if they co-vary with presentation time so that the final orientation remains the same. The overall movie duration can also be ruled out as a critical parameter suggesting that the results are predominantly driven by the final orientation of the object relative to the axis of translation.

\subsection{EXPERIMENT 3: EFFECT OF INITIAL ELLIPSE ORIENTATION}

The previous experiments have shown that the final orientation of the ellipse is a major parameter that determines if the percept is straight or curved. It is conceivable that the initial orientation may be similarly important. This experiment therefore investigated the impact of the initial orientation (Table 1D). The PSEs for four subjects are shown in Fig. 5 for three different initial orientations $\left(+10^{\circ},-35^{\circ}\right.$ and $\left.+90^{\circ}\right)$. 


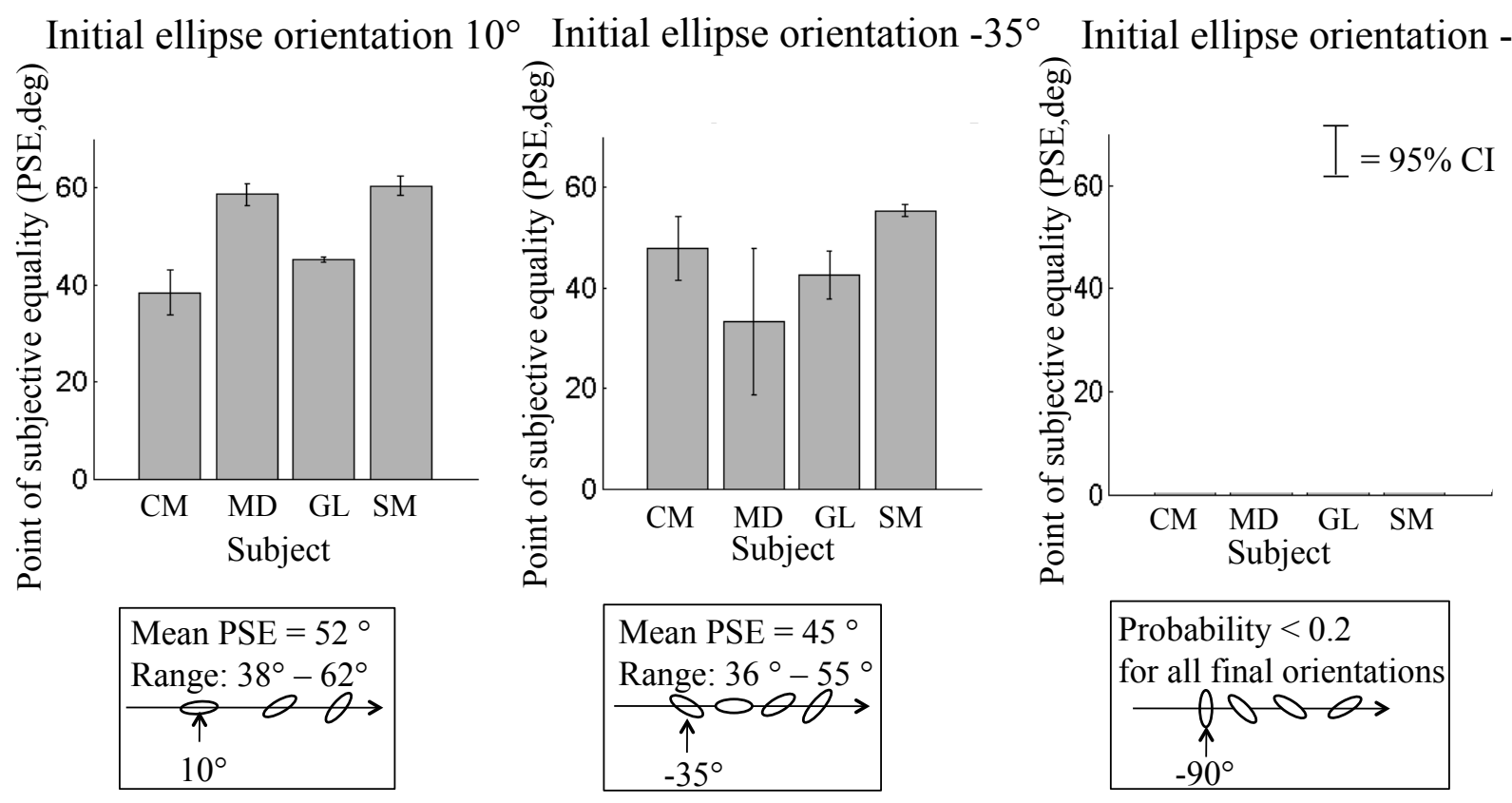

Figure 5: Effect of initial ellipse orientation. The PSEs for initial orientations of $+10^{\circ}\left(38-62^{\circ}\right.$, mean $\left.50.5^{\circ}\right)$ and $-35^{\circ}\left(36-55^{\circ}\right.$, mean $\left.45.0^{\circ}\right)$ are in the same range as in experiment 1 where the initial orientation was $-10 \mathrm{deg}$. When the initial orientation was $-90^{\circ}$, observers rarely reported a curved trajectory, irrespective of the final ellipse orientation tested, which made it impossible to calculate a PSE.

For an initial orientation of $+10^{\circ}$ (left), the average PSE was $50.5^{\circ}$ (range $28-62^{\circ}$ ) and a two-tailed $t$-test $(\mathrm{t}=0.81, \mathrm{df}=5.69, p=0.45)$ confirmed that this did not significantly differ from experiment 1 , where the initial orientation was $-10^{\circ}$. This is also the case for an initial orientation of $-35^{\circ}$ (centre; average $\mathrm{PSE}=45.0^{\circ}$, range $36-55^{\circ} ; \mathrm{t}=0.06, \mathrm{df}=7.35, p=0.95$ ). For an initial orientation of $-90^{\circ}$ (right), where the ellipse starts with a vertical orientation, observers did not typically perceive curved trajectories. Instead, the percept was dominated by an ellipse translating linearly along the horizontal. This further confirms the earlier observation that the presentation time does not have a major impact on the PSE. 
Taken together, as long as the orientation of the ellipse stays within about $\pm 35-45^{\circ}$, relative to the axis of translation, observers report a perception of a curved trajectory. Varying the initial or final orientations within that range appears to only have a minor effect on the percept. If, however, either the start or the final orientation is outside this range, observers predominantly perceive a linear trajectory.

\subsection{EXPERIMENT 4: EFFECT OF SHAPE: ASPECT RATIO}

The elongation of an ellipse (and therefore the prominence of its orientation) can be manipulated by varying its aspect ratio. If perception depends strongly on the ellipse's orientation, the illusionary percept might be enhanced by increasing the ellipse's aspect ratios (with a line being the extreme case) and gradually diminish as the shape becomes more circular. To investigate the effect of aspect ratio, the initial and final ellipse orientation, as well as the translational and rotational speeds, were fixed (Table $1 \mathrm{E})$. The particular values were chosen as they gave a strong percept of a curved trajectory in the earlier experiments.

Different shapes (aspect ratios) were randomly inter-mixed within an experimental block. The results (Fig. 6) suggest that the likelihood for a curved trajectory increases with increasing aspect ratio (i.e. the thinner the ellipse the more likely it is perceived on a curved path). For aspect ratios above approximately 1.5 , the predominant percept is of a curved trajectory while a straight percept dominated for aspect ratios below 1.5 (fat ellipses). The PSEs, where each percept is equally likely, are at aspect ratios between 1.4 and 1.6. This suggests that shape, linked perhaps to object elongation, is an important factor. 


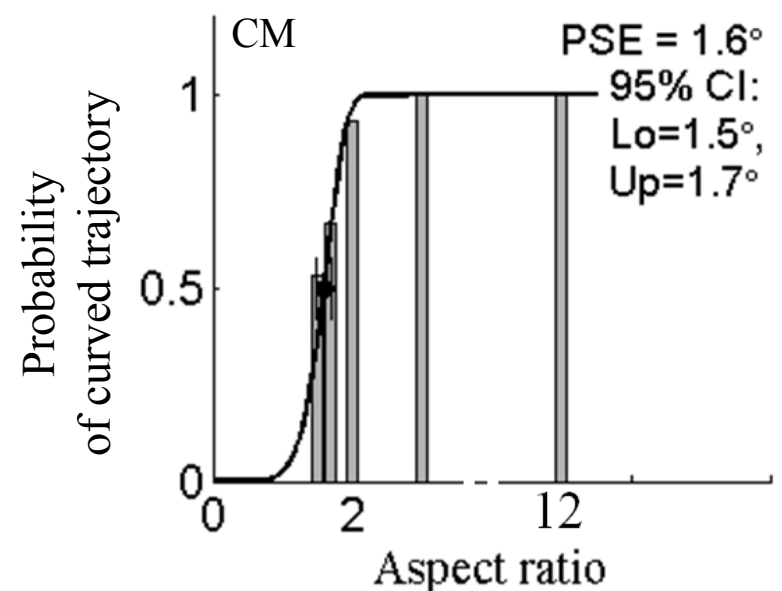

12

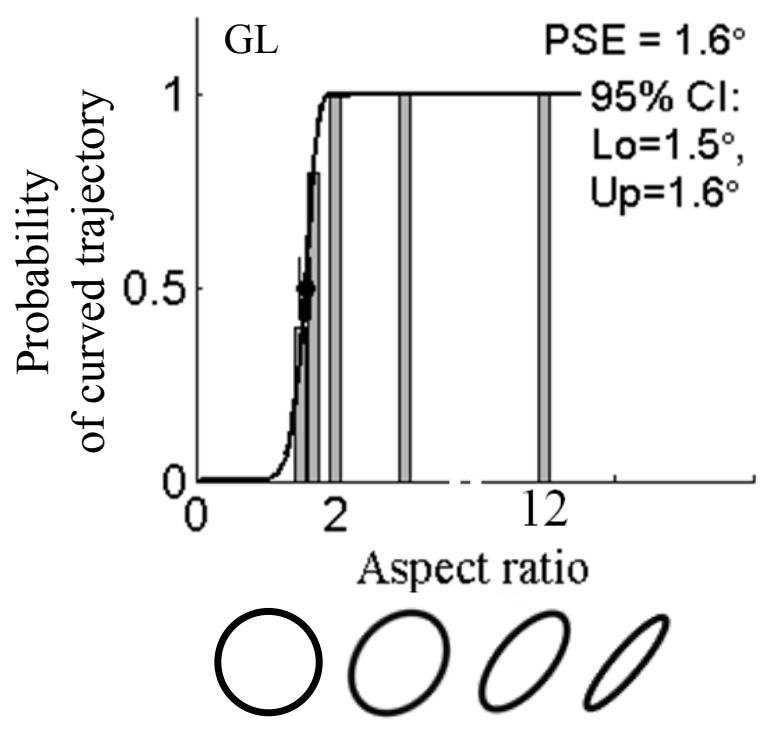

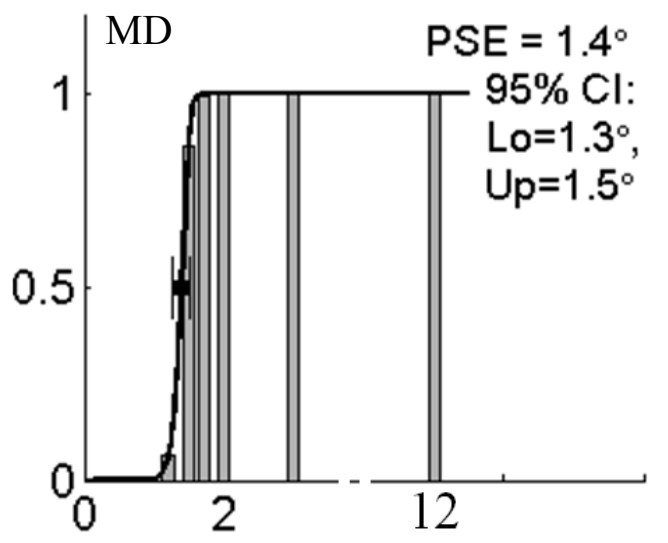

Aspect ratio

12

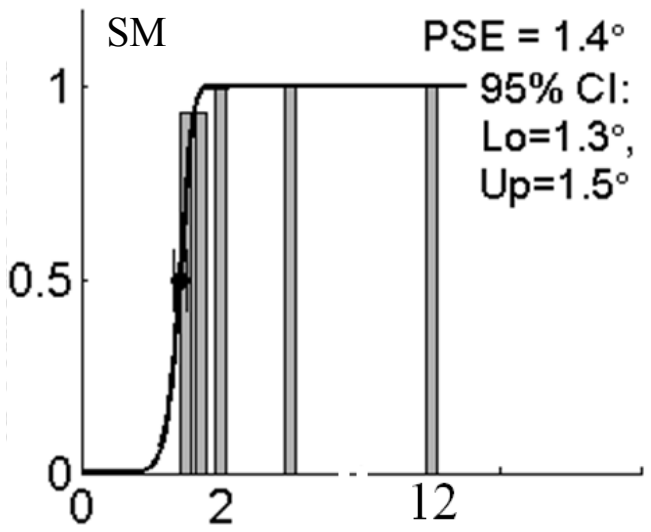

Aspect ratio

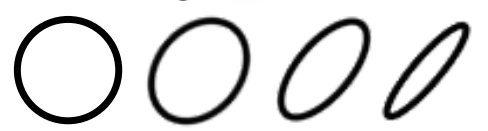

Figure 6: Probability of perceiving a curved trajectory as a function of the aspect ratio of the elliptical shapes for 4 observers. Seven aspect ratios were tested: 1, 1.2, 1.5, 1.7, 2, 3, and 12. The aspect ratio of the ellipses in all previous experiments was 3 . An aspect ratio of 1 corresponds to a circle for which rotation around its centre will be invisible to the observer. The resulting percept for a circle translating and 'rotating' is consequently that of it moving along a straight path. The average PSE is at an aspect ratio of 1.5 (range 1.4-1.6) that is observers are more likely to report a straight trajectory for lower aspect ratios and more likely to report a curved trajectory for higher ratios. 
One attribute of an elliptical shape that changes with aspect ratio is the maximum curvature at its tips. When the aspect ratio is decreased, the prominence of these features decreases as well. Studies have shown that features play an important role in perceiving rotational motion (Wallach, 1935; Weiss \& Adelson, 2000; Weiss et al., 2002; Caplovitz et al., 2006; Pack et al., 2004): de-emphasising the appearance of features minimizes the likelihood of perceiving rotation and observers become more likely to see rotating shapes as deforming non-rigidly.

It is conceivable that features may be an important factor in driving a curved versus a straight percept in our experiments. There are a number of ways in which features could influence perception. Features define the object's shape which is intrinsically linked to its axis of elongation. Features also carry veridical motion information and their path could, in theory be tracked and used to compute the perceived trajectory. In the experiments presented in the next section, we aimed to differentiate between these two by employing stimuli that contain identical features but differed in overall shape.

\subsection{EXPERIMENT 5: EFFECT OF SHAPE: TYPE OF OBJECT}

A rectangle, a square and a line were tested in order to observe how perception depends on object shape. The square lacks elongation, has the smallest aspect ratio (1), the highest number of symmetry axes (4) and also the smallest angular difference between adjacent symmetry axes $\left(45^{\circ}\right)$. Both rectangle and line have elongation and an angular difference of $90^{\circ}$ between their two axes of symmetry. Experimental parameters were those used in experiment 1 (see Table $1 \mathrm{~A}$ ) with the exception of the square for which a 
smaller number of final orientations $\left(+5^{\circ},+15^{\circ}\right.$ and $\left.+45^{\circ}\right)$ were selected based on pilot experiments.
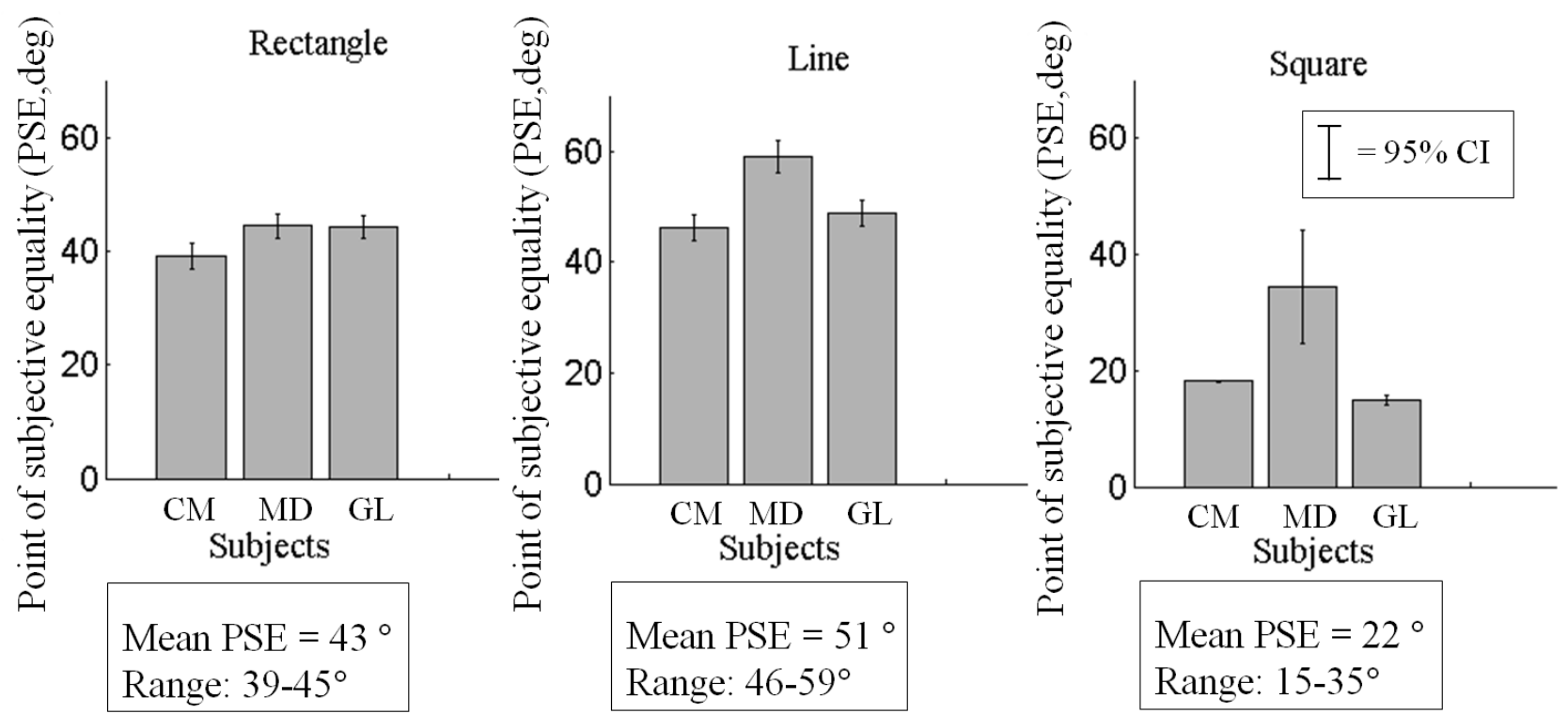

Figure 7: Probability of perceiving a curved trajectory for different objects: a rectangle, a line and a square. The PSEs for the rectangle $\left(42.9^{\circ}\right)$ is similar to that for the ellipse in the first experiment (45 $5^{\circ}$, Fig. 2). The PSE for the line is larger $\left(51.6^{\circ}\right)$, consistent with an increased probability of seeing curved paths for larger aspect ratios (experiment 4, Fig. 6). The PSE of the square is only about half the size of the ellipse and rectangle $\left(22.3^{\circ}\right)$.

The data (Fig. 7) show that the rectangle produces a percept (average PSE $=42.9^{\circ}$ ) similar to an ellipse $\left(45^{\circ}\right.$, experiment 1 ; Fig. 2$)$ with the same aspect ratio. A two sample, two-tailed $t$-test $(t=0.72, d f=16, p=0.48)$ indicates that there is no significant difference between the ellipse in experiment 1 and the rectangle here. This is consistent with observers relying on the orientation of the object along its elongation. It is further an indication that a difference in the position of salient features (ellipse: points of maximum curvature on the symmetry axis; rectangle: corners off the symmetry axis) does not result in different PSEs. The average PSE for a line is $51.6^{\circ}$, larger than that for the ellipse or 
rectangle and consistent with an increased probability of seeing curved path for larger aspect ratios (experiment 4, Fig. 6). A two sample two tailed t-test ( $\mathrm{t}=1.69$, df16, $p=0.11$ ) indicates that this difference is, however, not significant.

The square differs from the other shapes (ellipse, rectangle and line) as it lacks elongation. In experiment 4 , we observed that the probability of perceiving a curved trajectory for an ellipse reduced with decreasing aspect ratios. It was absent for circles that lack elongation (aspect ratio=1). If elongation, alone, was a pre-requisite for perceiving curved trajectories, one should not expect to see curved trajectories for squares. This is evidently not the case, although the likelihood is reduced. Comparing the data for the square to those for the rectangle and other elongated shapes shows that curved trajectories are seen over a smaller range of object orientations relative to translation axis, i.e. smaller PSEs. The average PSE of $22.3^{\circ}$ for squares are about half those of an elongated rectangle or ellipse $\left(\mathrm{PSE}=45^{\circ}\right)$. This difference is significant $(\mathrm{t}=3.25, \mathrm{df}=3.15, p=0.041)$.

The square contains the same number of features (corners) as the rectangle. If features play a determining role in driving the illusory curved percept then one should expect the data for a square and rectangle to be the same. As this is not the case, one can conclude that the mere presence of a feature is not driving the overall percept. In summary, these results point towards the critical role of an object's orientation that becomes more pronounced as the aspect ratio is increased (circle to ellipse; square to rectangle to line). According to this, the reason why a circle without elongation never gives rise to a curved percept whereas a square (also without elongation) sometimes does is because the circle, but not the square, lacks orientation. 


\subsection{EXPERIMENT 6: MEASURING THE SHAPE OF THE TRAJECTORY}

In the experiments so far, the investigation has focussed on the subjective report about whether linearly translating and rotating objects are perceived on a curved or straight trajectory. The purpose of this experiment was to determine the shape of the perceived trajectory in those circumstances where objects appear to follow a curved path. To do this, we measured the perceived direction of an object's motion at different instants in time (instantaneous directions of motion) and, assuming that these represent the tangent to the motion path, constructed the overall trajectory based on these measurements.

Perceived motion directions were measured using a 2AFC paradigm, where observers had to indicate if the motion of the object at the final point of a variable movie sequence was $\mathrm{CW}$ or $\mathrm{CCW}$ relative to the orientation of a static reference grating. The orientation of the grating was varied and the perceived direction of motion defined as the PSE extracted from a psychometric function fitted to the data. Two different ellipse configurations were used. The parameters of the first match those used in experiment 1 . The second configuration used an ellipse with the same aspect ratio but half the size. The PSEs are given in Table 2A for the large ellipse and in 2B for the smaller one. The data are for different movie durations and shown in the table as a function of the orientation of the ellipse in the last movie frame.

\begin{tabular}{|l|l|l|l|l|l|l|l|l|}
\hline & \multicolumn{7}{l}{ (A) Large Ellipse $\mathrm{a}=2, \mathrm{~b}=2 / 3$} \\
\hline Orientations & $0^{\circ}$ & $5^{\circ}$ & $10^{\circ}$ & $15^{\circ}$ & $20^{\circ}$ & $25^{\circ}$ & $30^{\circ}$ \\
\hline & Measured instantaneous directions of motion: \\
\hline CM & 1.2 & 6 & 10 & 14 & 22 & 19.9 & 32 \\
\hline GL & -6 & 0 & 13 & 22 & 20 & 11.9 & \\
\hline
\end{tabular}




\begin{tabular}{|l|l|l|l|l|l|l|l|l|}
\hline MD & -7 & 6 & 10 & 19 & 21 & 29 & 33 \\
\hline Median (mean) & -6 & 6 & 10 & 19 & 21 & 20 & $(32.5)$ \\
\hline & (B) Small Ellipse a=1, b=1/3 \\
\hline Orientations & $0^{\circ}$ & $5^{\circ}$ & $10^{\circ}$ & $15^{\circ}$ & $20^{\circ}$ & $25^{\circ}$ & $30^{\circ}$ \\
\hline CM & -1.1 & 7 & 11.8 & 13.2 & 23 & 24 & \\
\hline GL & -3.8 & -0.3 & 13.5 & 19.5 & 20.7 & 16 & \\
\hline MD & & 2 & 5.5 & & 20.5 & & 25.6 \\
\hline Median (mean) & $(-2.5)$ & 2 & 11.8 & $(16.4)$ & 21.4 & $(20)$ & $(25.6)$ \\
\hline
\end{tabular}

Table 2: Measured perceived directions of motion of a translating and rotating ellipse at different points during a movie. Data, for three observers and the median, are the PSEs (equally likely to report motion $\mathrm{CW}$ and $\mathrm{CCW}$ relative to a static grating) as a function of the orientation of the ellipse in the last movie frame (top row). The orientation of the ellipse, as well as the data, are relative to the physical axis of translation (horizontal). (A): Data for a large ellipse, identical to the one used in the first experiment. Data for a final orientation of $30^{\circ}$ are missing for one observer as he reported a straight trajectory for this condition. It is apparent that, to a first approximation, the perceived direction of motion follows the orientation of the ellipse. (B): Data for an ellipse of half the size as in (A). Different observers ran the experiment with different movie durations (final ellipse orientation). Where less than 3 data points are available, the typical performance is given as the mean rather than the median.

Euler integration was used to extrapolate trajectories from the data in Table 2. Fig. 8 shows the extracted horizontal and vertical position of the ellipse at different points in time, moving horizontally from -2DVA to the left of the centre at the start to +2DVA to the right at the end of the movie. The physical motion of the ellipses was linear translation along the horizontal while rotating so their centres remained on a horizontal line (i.e. at a vertical displacement of Odeg). Given the measured instantaneous directions of motion and assuming a constant horizontal velocity, the circles show the extrapolated perceived positions of the ellipse's centre at different points in time. The open circles indicate the position of the larger ellipse and the grey circles those for half that size, both calculated from the average PSE data. To provide an indication of the variability of the data, 
trajectories were also calculated for the maximum and minimum PSE values and are shown by the dashed lines. The black solid line shows a predicted trajectory under the assumption that perceived motion at different points in time was solely based on the static orientation of the ellipse.

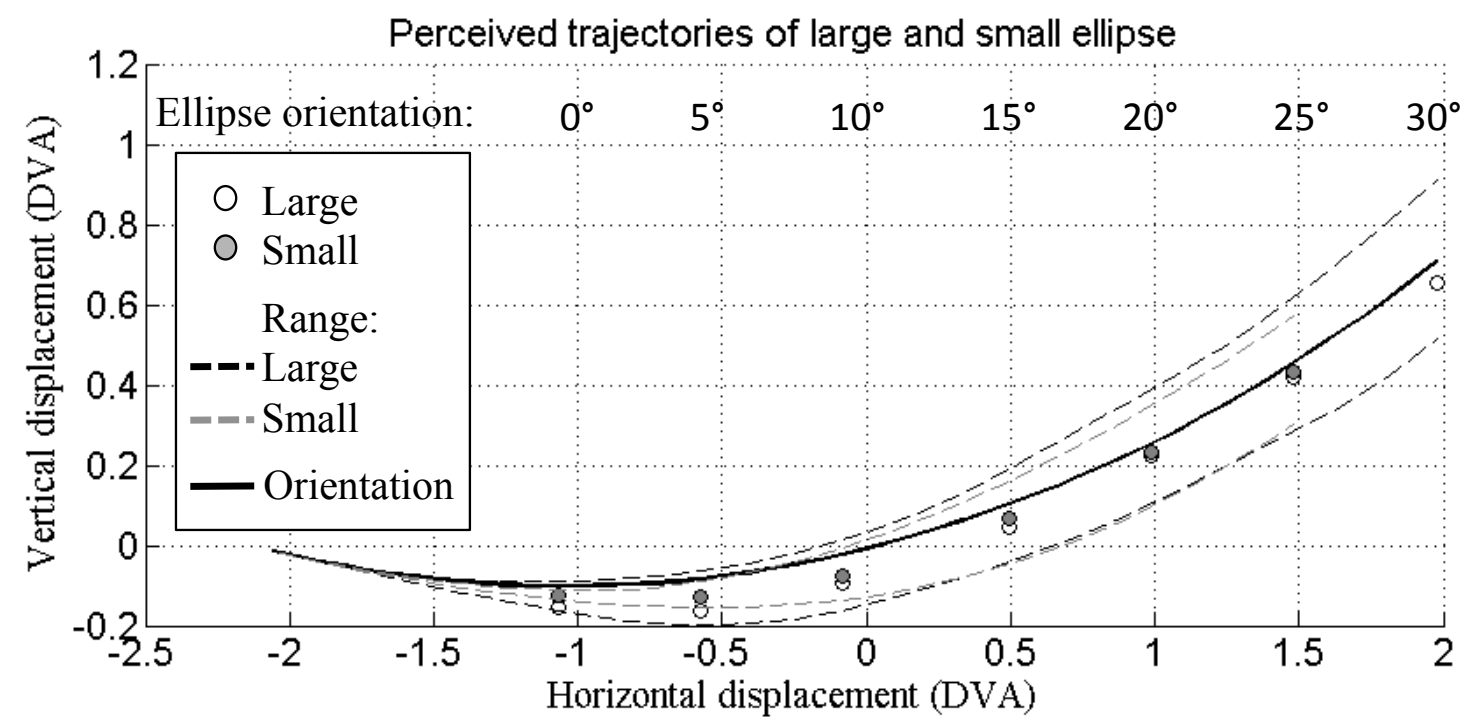

Figure 8: Horizontal and vertical positions extrapolated from the instantaneous direction of motion data in Table 2 for a small (filled circles) and large (open circles) ellipses. The motion sequence started at the left (-2deg relative to mid-point) and ended at the right $(+2 \mathrm{deg})$. The physical motion of the object was linear translation along the horizontal while rotating so the centre of the object remains on a horizontal line (e.g. at a vertical displacement of $0 \mathrm{deg}$ ). The circles show the calculated locations of the ellipses' perceived centre at different points in time, based on average data. The range is indicated by the dashed lines, which were created with the maximum and minimum PSEs. The black solid line shows a predicted trajectory under the assumption that perceived motion at different points in time was solely based on the static orientation of the ellipse. The trajectories for the two ellipse size are essentially overlapping and closely predicted by the orientation of the shapes.

The calculated positions for the two ellipse sizes are essentially the same. The extracted trajectories for the large and small ellipse are also close to the prediction based on the 
orientation of the objects. This suggests that the object's orientation plays a major role in the illusory curved percept.

\section{DISCUSSION}

This study aimed to investigate how perception of a translating object is affected by rotation. Observers were asked to judge the motion path of objects that rotated around their centroid while linearly translating. The expected percept, consistent with the actual dynamics used to generate the movies, is that of a translating and rotating object, akin to a tumbling rugby ball. We had, however, empirical reason to speculate that this may not be what observers perceive. Earlier studies have shown that, depending on a number of parameters, objects undergoing simple linear translation are not always seen to move veridically but may be biased towards their axis of orientation (Loffler \& Orbach, 2001; Magnussen et al., 2013; Morikawa, 1999). If this also applies to rotation, a change of orientation over time, the perceived direction of a translating and rotating object may continuously change following the changing orientation of the object. The resulting percept, an illusion, may therefore be that of an object moving along a curved path, similar to a car driving on a curved road. Our results show that both the veridical and nonveridical percept is possible for the same object. Which one of the two prevails depends on a number of factors. First, the object's orientation has to remain within a certain range relative to the axis of translation for the illusory, curved percept to dominate. If the orientation at any point of the movie sequence differs sufficiently from the axis of translation, the percept is that of linear translation with rotation. The angle at which the switch from curved to straight trajectory occurs is itself dependent on a number of factors that seem to relate to an object's elongation and, with it, the prominence of its orientation. 
Measuring the shape of the perceived illusory curved path shows that the perceived trajectory corresponds closely to the actual object orientation at different points during the movie sequence.

\section{Alternative motion decompositions and illusory percepts}

A number of studies have investigated how motion signals from different parts of a scene are combined when each piece of information on its own is ambiguous (the aperture problem), (Adelson \& Movshon, 1982; Ferrera \& Wilson, 1990; Wilson et al., 1992; Ben-Av \& Shiffrar, 1995; Castet \& Wuerger, 1997; Loffler \& Orbach, 1999, 2003a, 2003b). The presence of object features bypasses the necessity of combining ambiguous signals as they carry veridical information for objects that are translating rigidly in the fronto-parallel plane. 


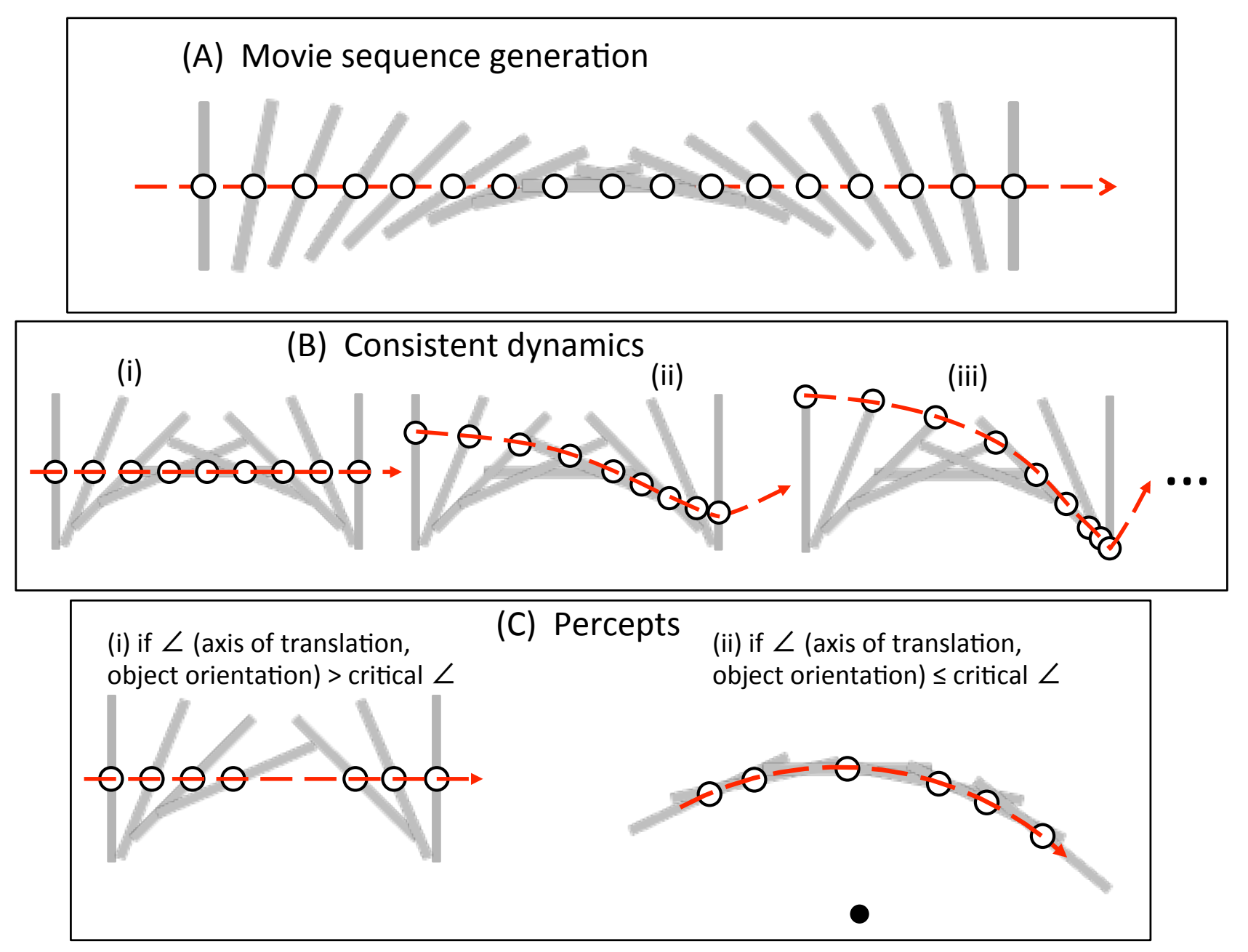

Figure 9: Alternative motion dynamics and percepts. (A) shows a sample movie sequence of a line, initially oriented vertically, translating linearly (red dashed line) from left to right while rotating around its centroid (white circle). All movie sequences in this study were created in this way: applying linear translation to the centroid of the objects and rotation around that point. (B) There are infinitely many different mathematical descriptions, physically identical, that can give rise to the same movie sequence in $(A)$. The three cases shown assume different centres of rotation. The rotational velocity is identical but the translation depends on the assumed centre of rotation. It is linear if the centre of rotation is the centroid of the line (i) but follows various cycloidal paths if it falls on peripheral points on the line (ii \& iii). The shape of the cycloid depends on the location of the assumed centre of rotation. (C) Under the experimental conditions tested, observers reported two distinct percepts. If the angle between the orientation of the object and its axis of translation at any point of the movie sequence fell outside a critical range, observers reported linear translation with rotation (i). If the angle remained sufficiently small, the percept changed to a curved trajectory in which the perceived, instantaneous directions of motion were close to the orientation of the object. The latter percept (ii) is consistent with object rotation around a peripheral point (black 
circle) but inconsistent with any of the physically valid descriptions (B) for the movie sequence in (A). This percept is an illusion (see text for further detail).

Such a unique description is, however, not available if an object is translating and rotating, even if it remains rigid and motion is constricted to the fronto-parallel plane ${ }^{3}$. An object moving with certain translational and rotational velocities is equally consistent with a variety of alternate velocity fields. Fig. 9 illustrates this. Fig. 9A shows an example of a translating and rotating line. Assume that the movie sequence was generated by a line translating linearly (red dashed line) and simultaneously rotating around its centroid (white circle), as was the case in our experiments. The decomposition of the object's motion into linear translation and rotation is not unique in the sense that an infinite number of different dynamics would result in the same movie sequence (e.g. Goldstein, 1980; Cutting \& Proffitt, 1982; Yang, Shimpi \& Purves, 2002). By assuming different, fixed centres of rotation on the object, the translational velocities can be adjusted to result in the same motion field (i.e. the same motion sequence). Fig. 9B shows three different combinations of rotation and translation that all result in the sequence shown in $(A)$. Different combinations differ in their translational trajectories and speeds but share the same rotational speed so that, e.g., a line that is initially oriented vertically will be oriented horizontally after the same amount of time has elapsed, regardless of the details of its translation. Case (i) shows the case of linear translation and rotation around the centre of

3 The non-uniqueness of descriptions of a translating and rotating object should not be conflated with either the issues of solving the undetermined problem of reconstructing a three-dimensional object from a two-dimensional retinal image, solving the undetermined problem of non-rigid motion of a featureless object (Ullman, 1979) or the ambiguities produced by the aperture problem when viewing a featureless grating through an aperture (Stumpf, 1911; Wallach, 1935). In all of these cases, there are multiple physical objects which could produce the same retinal image, while here multiple descriptions of the same physical object exist. 
the line. Rotation around the centroid is the only combination that results in linear translation. All other combinations contain centres of rotation (circles in Fig. 9) that translate on curved trajectories (cycloids, ii and iii). The shape of the trajectory therefore depends on the choice of the centre of rotation.

Hence, the percept of a curved trajectory, as observed in some of our experimental conditions, is not unique: both cycloidal paths in Fig. 9Bii and Biii are consistent with the description of a curved trajectory. A close inspection shows, however, that none of the possible cycloidal trajectories is consistent with our data in Fig. 8. Tangents (corresponding to instantaneous directions of motion of the object's centre) for cycloidal trajectories depend on the choice of the centre of rotation. For example, around the midpoint of the red trajectories in Fig. 9Bii and Biii, where the orientation of the line is close to horizontal and within the range where our observers reported curved trajectories, the tangents deviate more from horizontal as the centre of rotation is shifted away from the midpoint of the line. Irrespective of the shape of the cycloid (including the straight trajectory in Fig. 9Bi), there are always situations where the orientation of the line differs substantially from the tangent to the trajectory. Moreover, the angular difference between the trajectory and the orientation of the object changes over time. Hence, if observers perceived the motion sequence as a centre of rotation moving on a curved trajectory, they should report directions of motion that change over time relative to the object's orientation and should, at certain points, differ substantially from it. Our data (Fig. 8) do not support this. Subjects report "sailing" percepts with perceived directions of motion always remaining close to the orientation of the object. This fact argues against any of the cycloidal trajectories that would be consistent with the movie sequence and instead points to a different type of curved motion that is inconsistent with the movie. Fig. 9Cii illustrates 
such a percept that is consistent with the perceptual data but inconsistent with the actual motion. The perceived direction of the motion of the object changes over time corresponding to the change in orientation due to the rotation of the object. This percept is consistent with an object rotating around a centre that is distant from the object (black circle in Fig. 9Cii), similar to a bike travelling around a corner. This, or similar analogies (e.g. car on curved road, boat sailing on a curved path), were typically used when observers described their percept.

This, however, is an illusory percept. While any of the dynamics shown in Fig. 9B would be consistent with the movie sequence, the percept in Fig. 9Cii is inconsistent with it. The perceived position of the object that follows the curved path in Fig. 9Cii deviates from its actual position.

\section{Relation to previous studies}

The existence of multiple dynamics giving rise to the same motion sequence raises the interesting question as to what humans perceive when objects rotate and translate. This question has been addressed for the case of a line moving behind an aperture, so that the line ends were invisible (i.e. the terminators at the aperture border were extrinsic and the display lacked intrinsic terminators; Yang, Shimpi and Purves, 2002). Under specific circumstances, observers perceived a line that is rotating around a centre on the line while translating linearly as if it rotated around a centre that was distant from the line. The perceived centre was not fixed but seemed to change its location over time. The positions traced by the perceived centre followed the path of a cycloid. That study showed that this behaviour can be explained by considering all probabilities that one of many possible 
combination of translation and rotation causes the movement of the line. Using featureless lines, the situation is further complicated as a specific point on the line in one frame can correspond to any point on the line in a subsequent frame. The resulting probability distributions are therefore encapsulating all possible point-by-point correspondences for the given movie sequence. Faced with this uncertainty, the authors argued that motion perception is based on the maximum of the probability distribution that corresponds to a specific physical object motion giving rise to a particular motion sequence. For the conditions investigated in that study, this prediction captured the perceived centre of rotation well. Unlike our illusion, the perceived dynamics in their study are consistent with the image sequence, i.e. it is one of the many possible dynamics that result in the same movie.

Shiffrar and Pavel (1991) investigated the special case of the perception of featureless lines (behind an aperture) that are rotating without translation. Observers were asked to indicate the centre of rotation. The perceived centre of rotation was biased towards being on the line even when the line rotated around a point that was distant to it.

While these two studies provide insight into perception when confronted with translating and rotating objects, they both employed stimuli that lacked explicit object features. This results in a highly ambiguous situation even under the assumption of rigid motion in the fronto-parallel plane. With these set-ups, a point on the stimulus in one frame can correspond to any point on the stimulus in another frame, i.e. any motion component along the orientation of the contour cannot be determined (aperture problem; Stumpf, 1911; 
Wallach, 1935). In such circumstances, the visual system must solve the correspondence problem. This is not required when features are present as in our experiments.

To our knowledge, perception of a rotating and translating object where the entire object is visible and the feature signals (e.g. line terminators, tips of ellipses, corners of rectangles) are directly available has not been studied. Although perceptual transitions have been reported (Cutting \& Proffitt, 1982), a shift between two percepts (linear translation while rotating vs. curved trajectory) for the same stimulus is novel. A compelling demonstration of a perceptual transition that depends on the visual information available to observers is seen when lights are placed on an otherwise invisible rolling wheel (Proffitt, Cutting \& Steer, 1979). Presenting observers with an isolated single point on the circumference of such a rolling wheel results in a percept of a cycloidal path (similar to the red dashed lines in Fig. 9Bii \& iii). If another point is added at the centre of the wheel, the percept changes. The peripheral dot, tracing exactly the same path as before, is now perceived as rotating around the central, linearly translating dot. This demonstrates that perception for isolated dots can be consistent with the actual traced trajectory or with the decomposition of that trajectory into its translating and rotating components.

Our results show a perceptual shift even if the same visible information is available and an illusory path for one of the precepts. But, based on our results, we would expect the experimental set-up in Yang, Shimpi and Purves (2002) to reveal neither the transition nor the illusion. In their study, the initial orientation of the line relative to its translation at the starting point of the movie was always $>60 \mathrm{deg}$. As our experiments show (Figs. $2,3 \& 4$ ), 
observers generally perceive linear translation and rotation in circumstances where the orientation of the object differs from its axis of translation by that amount.

The more general case, where the visual system chooses between multiple physical configurations, was studied by Hildreth (1984). In order to solve the under-determined problem of deducting the 3D motion projected onto a 2D surface, Hildreth (1984) formulated a smoothness constraint. She argued that, when distinguishing between all possible velocity fields in 3D (including translation, rotation and deformation) that give rise to the same $2 \mathrm{D}$ projection, the one that minimises the overall motion variation in many cases, yields the veridical solution. In contrast to real deformation (e.g. Loffler \& Wilson, 2001), Hildreth showed that this analysis can result in the illusion of deformation as in the case of a nearly circular ellipse. When such an ellipse rotates rigidly, the smoothest velocity field indicates a non-rigid deformation, inconsistent with the object dynamics but consistent with perception (Wallach et al., 1956).

In other cases, perception is inconsistent with the optical stimulus. Retinal persistence has been suggested to be the cause of these illusions (Pomerantz, 1983; Burr, 2000; Burr \& Ross, 2002). Pomerantz (1983) analysed regions of different densities, which are inversely related to the velocity (i.e. slow movement results in high density), by superimposing successive frames of a motion sequence. The resulting density maps can explain illusory shape deformation as in the case of a line undergoing certain combinations of rotation and translation that makes it appear rubbery (the rubber pencil illusion). Such non-rigid illusions are very different from the illusory path seen here and it is unclear if an 
analysis based on a smoothness constraint or density maps can explain the transition between veridical and illusory percepts for the same object, depending on its orientation.

Other implementations of the idea of retinal persistence have been proposed (Burr, 2000; Burr \& Ross, 2002; Geisler, 1999). As the visual system sums information over time, individual image features on a moving object leave motion streaks. The traces of such streaks are consistent with the veridical object's directions of motion but inconsistent with the illusory curved trajectories and perceptual bi-stabilities seen here. Feature tracking may also be rejected as explanations for the particular shape of the perceived curved trajectory. Fig. 10 shows superimposed frames of two ellipses of different lengths, both rotating from $-10 \mathrm{deg}$ to $30 \mathrm{deg}$ relative to the horizontal while translating to the right. If feature tracking was responsible for the percept of the curved trajectory, its shape should change with ellipse size because the path, which connects the tip of the ellipse (the prominent features with maximum curvature), changes. In the case of a large ellipse (Fig. 10, left, blue line), these features follow a steeper path than when the ellipse is small (right, green line). Hence, if the trajectory was influenced by feature tracking, the percept of a curved trajectory should diminish with decreasing size and the perceived trajectory should become straighter, approaching the percept of translation on a straight line. This is inconsistent with the measured perceived trajectories, which are independent of ellipse size (Fig. 8). Hence, it is unclear if any of these proposals (smoothness constraint, density maps, motion streaks and feature tracking) can explain our data. 

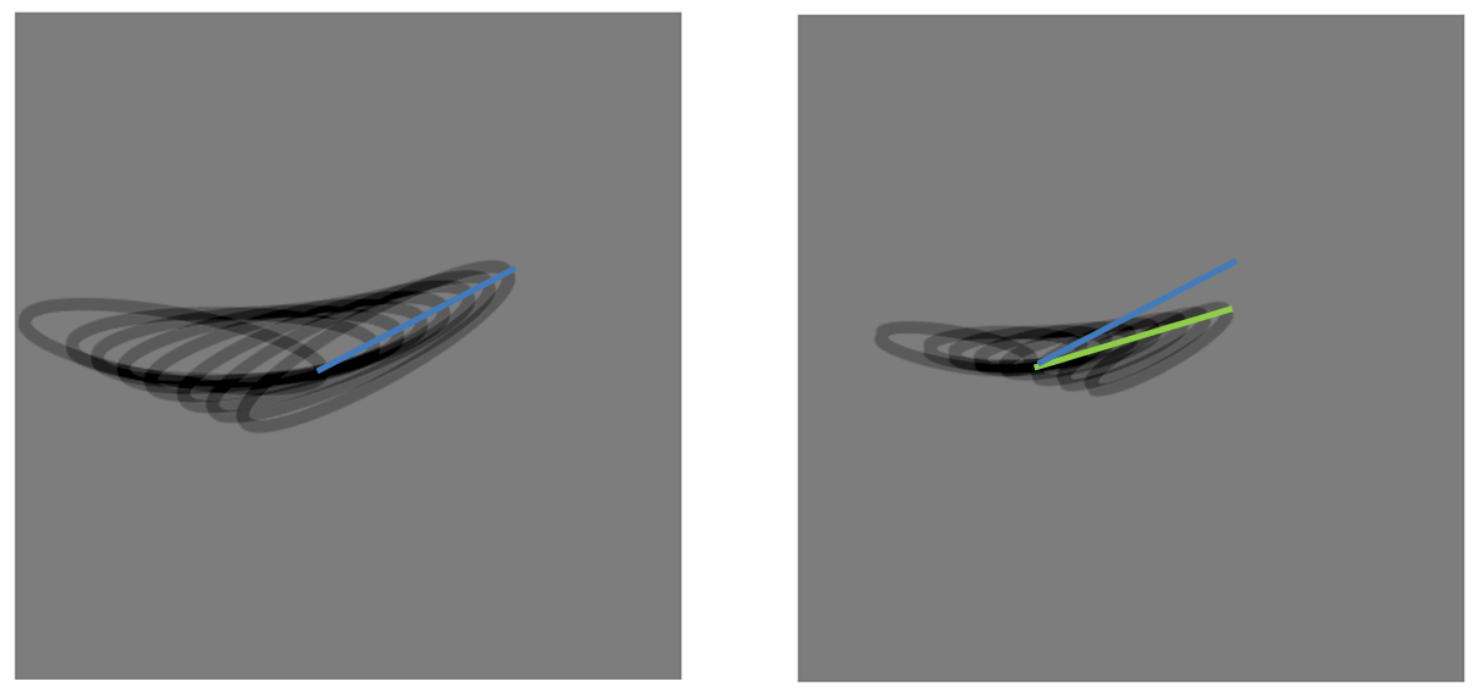

Figure 10: Feature tracking. The graphs show superimposed frames of two ellipses of different size, both rotating from $-10 \mathrm{deg}$ to $30 \mathrm{deg}$ while translating to the right. The blue and green lines show the paths that connect the position of the prominent features (tips) of the large and small ellipses, respectively. For the range of frames shown here, these paths are reasonably well approximated by straight lines and are shown as such for simplicity. For more extended sequences, they would trace the shape of a cycloid. The path depends on the size of the ellipse and is shallower for the smaller size (green, right). As perception is largely independent of size, feature tracking can be excluded as an explanation for the perceived trajectories.

One characteristic that remains unchanged when size is manipulated is object orientation. Changing the size of the ellipse changes the physical locations of the ellipse tips but not its orientation. The lack of dependence of the perceived trajectory on size therefore argues in favour of the orientation of the ellipse as the critical parameter. Our results therefore suggest that the percept is driven by orientation cues even when an abundance of motion information is present (including signals from multiple features and contour points). The perceived directions of motion are close to the orientation of the object. This is in agreement with our theory that observers tend to perceive motion along the orientation of an object (Magnussen et al, 2013). This preference can result in biases away from 
veridical motion when objects undergo simple translations (Magnussen et al, 2013; Morikawa, 1999). When objects rotate during translation so that their orientations change over time, this, under certain circumstances, can result in an illusory percept of an object translating on a curved trajectory. It would be interesting to see if this is driven by the real or perceived orientation of the object (Kennedy, Orbach \& Loffler, 2006 \& 2008).

In these cases, the resulting percept is consistent with rotation around a centre that is distant from the object with the perceived curved trajectory tangent to its orientation. This general percept is in line with many natural objects such as an animal running on a curved path. There, the orientation of the object changes to stay tangential to the curved trajectory and the rotation is therefore constrained by the translation. It is possible that we tend to describe and, perhaps, perceive object motion in ways which are consistent with descriptively sparse special cases that we observe in nature such as: objects tumbling along simple trajectories (spinning car) or objects "sailing" along trajectories consistent with their orientations. Our results might reflect a general preference for perceiving objects rotating around a point off the object so that their orientation remains tangent to the trajectory. In the circumstances shown here, however, this preference results in an illusion. 


\section{REFERENCES}

Adelson, E. H. \& Movshon, J. A. (1982) Phenomenal coherence of moving visual patterns. Nature 300, 523-525.

Ben-Av, M.B \& Shiffrar, M. (1995). Disambiguating velocity estimates across image space. Vision research 35, 2889-2895.

Burr, D. (2000). Motion vision: Are 'speed lines' used in human visual motion? Current Biology 10, R440-R443.

Burr, D. C. \& Ross, J. (2002). Direct evidence that "speedlines" influence motion mechanisms. Journal of Neuroscience 22, 8661-8664.

Caplovitz, G. P., Hsieh, P. J. \& Tse, P. U. (2006). Mechanisms underlying the perceived angular velocity of a rigidly rotating object. Vision Research 46, 2877-2893.

Castet, E \& Wuerger, S (1997). Perception of moving lines: interactions between local perpendicular signals and 2D motion signals. Vision research 37, 705-720.

Cutting, J. E. \& Proffitt, D. R. (1982). The minimum principle and the perception of absolute, common, and relative motions. Cognitive Psychology 14, 211-246.

Ferrera, V. P. \& Wilson, H. R. (1990) Perceived direction of moving two-dimensional patterns. Vision Research 30, 273-287.

Geisler, W. S. (1999). Motion streaks provide a spatial code for motion direction. Nature 400, 6569.

Goldstein, H. (1980). Classical mechanics. Addison-Wesley Publishing Company.

Grossberg, S \& Mingolla, E. (1993). Neural dynamics of motion perception: Direction fields, apertures, and resonant grouping. Perception and Psychophysics, 53, 243-278

Hildreth, E. C. (1984). Computations underlying the measurement of visual motion. Artificial Intelligence 23, 309-354.

Kennedy, G., Orbach, H.S., Gordon, G.E. \& Loffler, G., (2008), Judging the shape of moving objects: Discriminating dynamic angles. Journal of Vision 8/13/9, 1-13.

Kennedy, G., Orbach, H.S., Loffler, G. (2008), Global shape versus local feature: an angle illusion. Vision Research 48/11, 1281-1289

Kennedy, G., Orbach, H.S., Loffler, G. (2006), Effects of global shape on angle discrimination. Vision Research 46/8-9, 1530-1539

Kim, J. \& Wilson, H. R. (1994). Dependence of plaid motion coherence on component grating directions (VOL 33, PG 2479, 1993). Vision Research 34, 973-973.

Loffler, G \& Orbach, H.S. (1999). Computing feature motion without feature detectors: a model for terminator motion without end-stopped cells. Vision research 39, 859-871. 
Loffler, G \& Orbach, H.S. (2001) Anisotropy in judging the absolute direction of motion. Vision research. 41, 3677-3692.

Loffler, G \& Wilson H.R., (2001), Detecting shape deformation of moving patterns. Vision Research, 41/8, 991-1005.

Loffler, G \& Orbach, H.S. (2003a). Factors affecting motion integration. Journal of the Optical Society of America a-Optics Image Science and Vision 20, 1461-1471.

Loffler, G \& Orbach, H.S. (2003b).Modeling integration of motion signals across space. Journal of the Optical Society of America a-Optics Image Science and Vision 20, 1472-1489.

Lorenceau, J \& Shiffrar,M (1992). The influence of terminators on motion integration across space. Vision Research 32, 263 -273.

Magnussen, C.M, Orbach, H.S \& Loffler, G. (2013). Motion trajectories and object properties influence perceived direction of motion. Vision Research 91,21-35.

Morikawa,K (1999). Symmetry and elongation of objects influence perceived diretion of translational motion.

Nakayama,K \& Silverman, G.H (1988). The aperture problem 2. Spatial integration of velocity information along contours. Vision Research 28, 747-753.

Pack, C.C, Gartland, A.J \& Born, R.T. (2004). Integration of contour and terminator signals in visual area MT of alert macaque. Journal of Neuroscience 24, 3268-3280.

Pomerantz, J. (1979). Rubber pencil illusion explained. Bulletin of the Psychonomic Society 14 , 251-251.

Pelli, D. G. (1997). The VideoToolbox software for visual psychophysics: Transforming numbers into movies. Spatial Vision 10, 437-442.

Pomerantz, J. R. (1983). The rubber pencil illusion. Perception \& Psychophysics 33, 365-368.

Prins, N \& Kingdom, F. A. A. (2009) Palamedes: Matlab routines for analyzing psychophysical data. http://www.palamedestoolbox.org

Proffitt, D. R. \& Cutting, J. E. (1980). An invariant for wheel-generated motions and the logic of its determination. Perception 9, 435-449.

Proffitt, D. R., Cutting, J. E. \& Stier, D. M. (1979). Perception of wheel-generated motions. Journal of Experimental Psychology-Human Perception and Performance 5, 289-302.

Quick, R. F. (1974). A vector-magnitude model of contrast detection. Kybernetik 16, 65-67.

Shiffrar, M. \& Pavel, M. (1991). Percept of rigid motion within and across apertures. Journal of Experimental Psychology-Human Perception and Performance 17, 749-761.

Stumpf. (1911). The histology of neurohypophysis. Virchows Archiv Fur Pathologische Anatomie Und Physiologie Und Fur Klinische Medizin 206, 70-79.

Ullman, S. (1979a). Interpretation of structure from motion. Proceedings of the Royal Society of London Series B-Biological Sciences 203, 405-426. 
Wallach, H. (1935). Über visuell wahrgenommene Bewegungsrichtung. Psychologische Forschung, 20, 325-380.

Wallach, H., Weisz, A. and Adams, P.A., Circles and derived figures in rotation, Amer. J. Psychol. 69 (1956) 48-59.

Weiss, Y. \& Adelson, E. H. (2000). Adventures with gelatinous ellipses - constraints on models of human motion analysis. Perception 29, 543-566.

Weiss, Y., Simoncelli, E. P. \& Adelson, E. H. (2002). Motion illusions as optimal percepts. Nature Neuroscience 5, 598-604.

Wilson, H. R., Ferrera, V. P. \& Yo, C. (1992) A psychophysically motivated model for twodimensional motion perception. Visual Neuroscience 9, 79-97.

Yang, Z. Y., Shimpi, A. \& Purves, D. (2002). Perception of objects that are translating and rotating. Perception 31, 925-942. 